\title{
Visual pursuit response in the severe disorder of consciousness: modulation by the central autonomic system and a predictive model
}

\author{
Francesco Riganello ${ }^{1}$, Maria D Cortese ${ }^{1}$, Giuliano Dolce ${ }^{1}$ and Walter G Sannita $2,3^{*}$
}

\begin{abstract}
Background: A visual pursuit response is reportedly observed in 20-30\% of subjects in vegetative state (VS/UWS) and predicts better outcome; it is a key marker of evolution into the minimally conscious state (MCS). The probability of observing a positive response, however, has proven variable during the day, with comparable timing of the minima and maxima in VS/UWS and MCS. We verified if measures of sympathetic/parasympathetic balance are possible independent variables on which the occurrence of a pursuit response could depend and be predicted.

Methods: Fourteen subjects in VS/UWS and sixteen in MCS for more than one year were studied. A mirror was used to test the pursuit response for a total 231 useful trials. Non-invasive measures of the sympathetic/parasympathetic functional state (Heart rate variability descriptors nuLF and peakLF) used in the study of responsiveness in VS/UWS and MCS subjects were recorded and processed by descriptive statistics and advanced Support Vector Machine (SVM).
\end{abstract}

Results: A pursuit response was observed in $33 \%$ and $78.2 \%$ of subjects in VS or MCS, respectively. Incidence was higher at HRV nuLF values in the 20-60 range and peakLF values at $0.06-0.12 \mathrm{~Hz}(76.6 \%)$ and at nuLF values in the 10-60 range and peakLF values at $0.05-0.10 \mathrm{~Hz}(80.7 \%)$ in the VS and MCS, respectively. The SVM generated model confirmed the results in the training leave one out and 10 fold cross validation tests (81\% and $81.4 \%$ ).

Conclusion: The pursuit response incidence depends to a relevant extent on the sympathetic/parasympathetic balance and autonomic functional state. Extensive monitoring appears advisable.

Keywords: Disorder of consciousness, Visual pursuit response, Vegetative state, Minimally conscious state, Central autonomic system, Heart rate variability, Support vector machine

\section{Background}

A visual pursuit response (the "pursuit eye movement or sustained fixation that occurs in direct response to moving or salient stimuli") is reportedly observed in $\sim 20-30 \%$ of the severely brain damaged subjects otherwise characterized by all clinical features of the vegetative state (VS; also referred to as the unresponsive wakefulness syndrome or UWS) [1] and predicts a favorable outcome with accuracy higher than $70 \%$. The response is also a key marker of evolution into the minimally conscious state (MCS; a condition with evidence of partially recovered

\footnotetext{
* Correspondence: wgs@dism.unige.it

${ }^{2}$ Department of Neuroscience, Ophthalmology and Genetics, University of Genova, 3, Largo P. Daneo, 16132 Genova, Italy

${ }^{3}$ Department of Psychiatry, State University of New York, Stony Brook, NY, USA

Full list of author information is available at the end of the article
}

awareness of self or environment, in which it is observed in $\sim 70-80 \%$ of subjects) and is a major item of the revised Coma Recovery Scale $[1,2]$.

The response incidence in VS/UWS and MCS is thought to further blur boundaries between these conditions and to reduce diagnostic accuracy, with an estimated misdiagnosis rate up to $25-45 \%$ [3]. Multiple testing on subjects in VS/ UWS or MCS (6 tests/subject/day) confirmed a varying incidence of the pursuit response during the day, with maxima at 10.30 am and $3.00 \mathrm{pm}$, no response at postprandial time, and an overall chance of observing it at least once per day of $\sim 33 \%$ and $\sim 62 \%$ in the VS/UWS and MCS, respectively [4]. The occurrence of a positive pursuit response thus appears conditional to changes in the functional brain state that may occur spontaneously

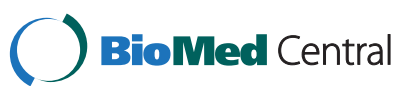


or be induced by a variety of possible neuronal or nonneuronal factors, and may question the sign reliability and pathophysiological meaning.

Replicable changes in the sympathetic/parasympathetic functional state in response to simple emotional or complex sensory stimulus conditions have been described in VS/UWS and MCS subjects [5-7] for references. The observation is in the same line of evidence with neuroimaging studies documenting in these patients a residual responsiveness, i.e. stimulus- or condition-related regional brain activation $[8,9]$. Measures of the sympathetic/ parasympathetic balance thus appear to be possible independent variables against which the occurrence of a pursuit response be tested. To this end, we have investigated in VS/UWS and MCS subjects the correlation between the presence/absence of a pursuit response and measures of heart rate variability (HRV). HRV (i.e. the heart rate fluctuation around the mean value over the time sample) is regarded today as a reliable index of the sympathetic/parasympathetic interplay and intrinsic influence on heart rate; it is deemed also applicable in the description of the brain and autonomic system interaction and functional organization in homeostasis and homeostatic responses both in awake subjects and in the severe disorder of consciousness [10-13].

\section{Methods}

\section{Subjects}

Fourteen subjects diagnosed as being in VS/UWS (10 males, age range: $27-73$ yrs., mean: $45 \pm 17$ yrs.) and sixteen in
MCS (9 males, age range: $33-77$ yrs., mean: $55.2 \pm 16.3$ yrs.) for more than one year according to the current clinical criteria and established evaluation scales were studied [2]. Scores were lower than 25 at the Loewenstein Scale [14], 2 at the Level of Cognitive Function scale (LCF) [15] and higher than 21 at the Disability Rating Scale [16] in the VS/UWS; LCF scores were 3 for subjects in the MCS. The global score of the revised Coma Recovery Scale (CRS-r) [17] was lower than 8 in VS/UWS subjects and between 10 and 14 in the MCS. Subjects clinically unstable, under treatment with neuroactive drugs or beta-blockers or with concurrent systemic disorders or evidence of recurrent pain were not admitted to the study. Demographics and the relevant clinical information are summarized in Table 1.

The "Independent ethical committee ASP" of the public health administration of Crotone (Italy) approved the study and the experimental procedures. The ethical principles of the Declaration of Helsinki (1964) concerning human experimentation were carefully followed throughout the study. The patients' relatives or caregivers were informed in full detail about the study and procedures and gave their written consent.

\section{Stimulus conditions and experimental procedure}

Subjects were nursed before 9.00 am compliant to the unit rules and tested for a visual pursuit response at $9.30-10.30 \mathrm{am}$, i.e. at the time of the day when the response incidence had proven highest in a previous study [4]. They were comfortably sitting on armchair

Table 1 Summary of the patients' demographics and clinical condition

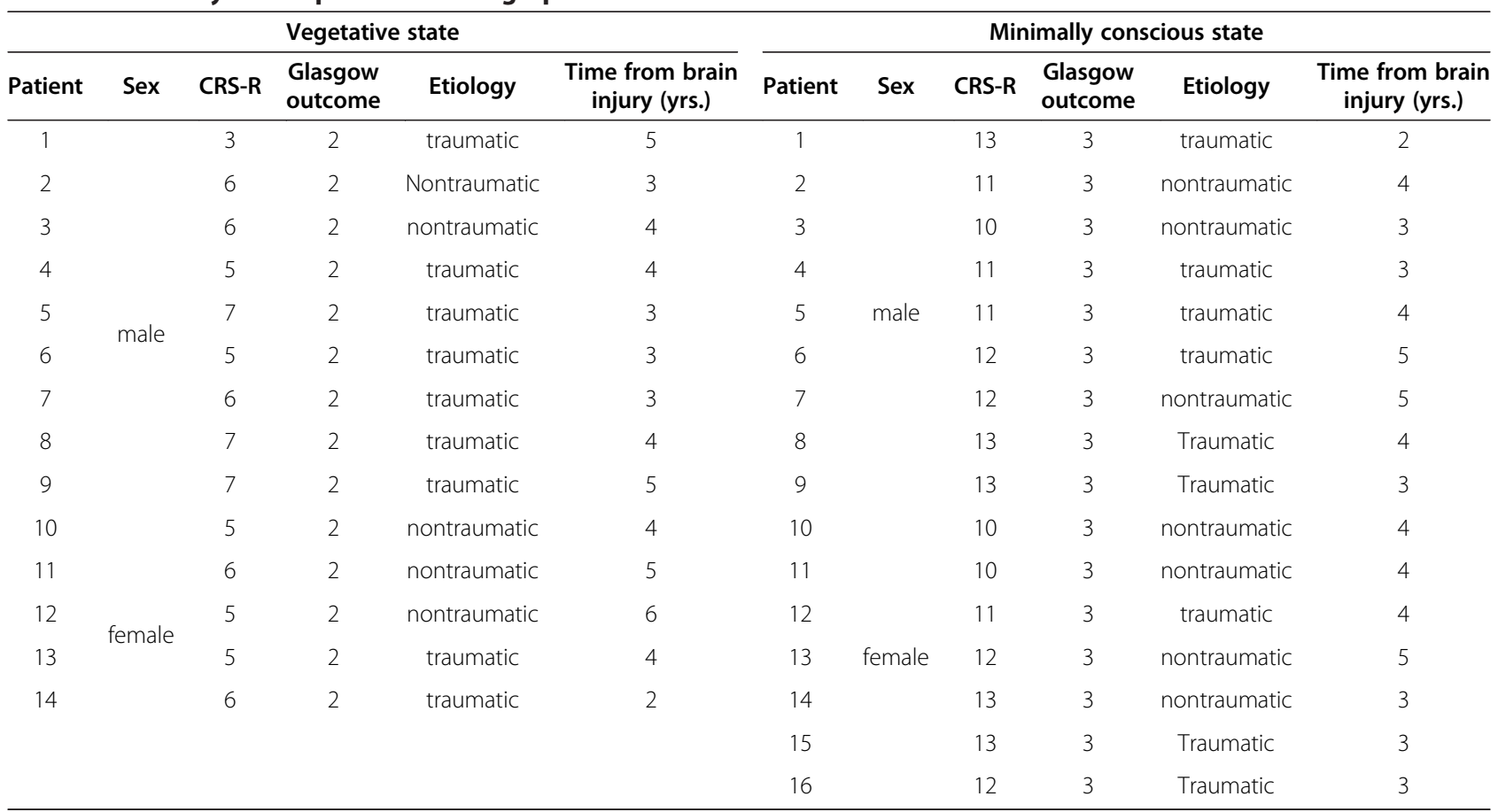


with constant $24^{\circ} \mathrm{C}$ ambient temperature in the absence of transient noise. A round mirror $(12 \mathrm{~cm}$ in diameter) was moved slowly in front of the subjects in the horizontal and vertical planes for $45^{\circ}$ (right, left, up, down) in order to obtain a visual pursuit response. The procedure was replicated in each direction and randomized. Subjects were tested when their eyes were open and no sign or sleep or drowsiness could be detected upon observation; in no case sensory or noxious stimuli were administered to stimulate patients into wakefulness. The test was performed by an expert neuropsychologist familial to all subjects; it was ranked positive for a consistent response when the patient's eyes followed the mirror without loss of fixation. The procedure was in accordance with the CRS-r guidelines [17]. Nine (3.75\%) of the 240 stimulus conditions were discarded because of artifact mostly due to sudden movements such as cough or grimace, with a total of 231 useful trials.

\section{Heart rate variability: data collection}

The heart rate was recorded continuously before (5 min., baseline) and during each testing procedure by means of a photopletismograph positioned on the middle finger of the left hand and interfaced with a Nexus 10 commercial data acquisition system (www.mindmedia.nl); sampling was at $128 \mathrm{~Hz}$, resolution at $24 \mathrm{bit}$, the average recording time during testing $224 \pm 3 \mathrm{sec}$. These recording procedure and sampling rate were favored to minimize the subjects' discomfort and the equipment interference; although not as precise as high-rate sampled EKG, it has proven reliable in studies on healthy [18] and in VS/UWS and MCS subjects [19] and is in agreement with the Task Force of European Society of Cardiology and the North American Society of Pacing and Electrophysiology guidelines [20]. The tachogram (i.e. the series of consecutive intervals between heart beats) was analyzed in the time and frequency domains by means of the Biotratace + (www.mindmedia.nl) and Kubios dedicated software for HRV measurements [21]. Non- parametric Fast Fourier Transform and Welch spectrum analyses were performed in the 0.01 to $0.5 \mathrm{~Hz}$ interval with $0.001 \mathrm{~Hz}$ resolution. The spectral descriptors in three frequency ranges (very low frequency [VLF]: $0.01-0.04 \mathrm{~Hz}$; low frequency [LF]: $0.04-0.15 \mathrm{~Hz}$; and high frequency [HF]: $0.15-0.5 \mathrm{~Hz}$ ) were computed [20].

\section{Heart rate variability: selection of descriptors}

Previous studies applying advanced data mining tools to sort out consistent trends or associations in large datasets [22,23] have identified the normalized LF index $(n u L F)$ and the LF peak $(p k L F)$ as reliable descriptors of sympathetic/parasympathetic function in the VS/UWS and MCS $[5,6,13,19]$. These parameters are known to describe the control of cardiovascular function in adaptive behavior and the interaction between excitatory and inhibitory autonomic control mechanisms and were thus entered into the statistical analyses. Specifically, $n u L F$ is deemed indicative of sympathovagal balance [24]. The $0.1 \mathrm{~Hz}$ component $L F$ (pkLF) describe the sympathetic/ parasympathetic interplay mediated by baroreflex and has been related to changes in arousal and to emotional responses [25].

\section{Descriptive statistics and modeling}

The HRV descriptors of the VS/UWS and MCS subgroups were compared in baseline to exclude differences depending on the clinical condition already at rest (Mann-Whitney U test). The correlation of the presence/ absence of a visual pursuit response with each HRV descriptor was also tested by the Mann-Whitney test. The probability of observing a visual pursuit response was estimated as the relative frequency of response for each subject vs. each HRV descriptor ( $n u L F$, peak $p k L F$ ). The dataset was also analyzed by feature selection technique and Support Vector Machine classifier in order to model the probability of observing a visual pursuit response on the basis of the actual HRV measurements. Feature selection is used in predictive data techniques whereby relevant features (variables) are to be selected to build robust learning models and develop predictive models for the event of interest based on a large number of predictors [26]. Support Vector Machine (SVM) is a machine learning methodology of widespread use in classification, regression and ranking $[27,28]$. A state of art classification method with high accuracy and flexibility, SVM is used in bioinformatics and other disciplines to model data of varying source and meaning [28]; applicability in HRV measures processing has been documented [29]. In this study, SVM was used to develop a model able to predict target data values (presence or absence of a visual pursuit response) to which specific attributes (the HRV descriptors) could be related at the different steps of the training set for classification. The Generalization levels of correct classifications (model) were tested and validated by the Leave One Out and Ten Fold Cross Validation tests [30]. The Radial Basis Function (RBF) was selected as the kernel function to be used to solve the nonlinear problem [31]. The model sensitivity and specificity were estimated as the proportions of actual presence or absence of visual pursuit correctly classified. The method conditions of application allow reasonably exclude a "double dipping" bias due to the use of the same data for selection and selective analysis and possibly resulting in distorted descriptive statistics and invalid inference.

\section{Results}

A visual pursuit response was observed in 33\% and $78.2 \%$ of subjects in VS/UWS or MCS, respectively. The 
distributions of the HRV descriptors in the subgroups of VS/UWS or MCS subjects did not differ in baseline (Mann Whitney U test $\mathrm{z}>-0.665, \mathrm{p}>0.5$ ) (Figure 1).

The observation of a visual pursuit response depended on the nuLF and $p k L F$ values in both patients' subgroups. The positive pursuit responses of the VS/UWS patients were clustered (76.6\%) at $p k L F$ values between 0.06 and $0.12 \mathrm{~Hz}$ and $n u L F$ values between 20 and 60; in MCS, the positive responses were clustered (80.7\%) at $0.05-0.10 \mathrm{~Hz} p k L F$ values and $n u L F$ values between 10 and 60 (Figure 2). The estimated probability of observing a response was described by polynomial (cubic) curves, with a maximum peak of response in both VS/UWS and MCS subgroups at $n u L F$ values between 20 and $40\left(\mathrm{R}^{2}\right.$ : 0.918 and 0.854 for the VS/UWS and MCS, respectively) and $p k L F$ values at $0.06-0.08 \mathrm{~Hz}\left(\mathrm{R}^{2}: 0.999\right.$ and 0.937 for the VS/UWS and MCS, respectively) (Figure 2). The $p k L F$ values interval at which positive responses clustered was comparable in the VS/UWS and MCS subgroups $\left(\chi^{2}=2.811, \mathrm{p}=0.58\right)$, while that of $n u L F$ was wider in the MCS subgroup $\left(\chi^{2}=14.593, \mathrm{p}=0.005\right)$.

The SVM tool modeled a nonlinear function describing the relationship of the HRV descriptors $p k L F$ and $n u L F$ with the presence or absence of a pursuit response. In the training test, the SVM-generated model gave percentages of correct classification of $88.8 \%$ and $86.9 \%$ in MCS and VS subgroups, respectively (Table 2). The good correct classification was confirmed at both validation tests (Table 2).

\section{Discussion}

Converging neuroimaging, electrophysiological and clinical observations provide evidence of direct/indirect functional links between HRV measures, autonomic control, and brain activity in structures that are involved also in attention and conscious processes [32,33]. In this regards, a model network (The Central Autonomic Network;
CAN) has been proposed, in which the anterior cingulate cortex and its projections to the prefrontal cortex, amigdala, hypothalamus and brainsteam are involved in the modulation of autonomic output in response to pain and emotional or behavioral stimulus conditions [13]. Neuroimaging studies in healthy volunteers have shown that the visual pursuit response depends on activation in structures of the anterior and posterior midline (mesiofrontal and precuneal cortices) [34] which are metabolically impaired in the brain damaged with severe disorder of consciousness who are incapable of sustained visual pursuit [35]. The re-appearance of a positive response in these subjects is thus regarded as indicative of functional upgrading in the subject's clinical condition and substantial recuperation of the corticocortical and brainstem-cortex interaction that is thought to be anatomically/functionally interfered with in the VS/UWS and MCS $[2,8,9,35,36]$. The observed higher incidence of a pursuit response at values of HRV descriptors deemed indicative of sympathetic/parasympathetic balance suggests that its within-day incidence (and, by extension, the subject's variability in brain responsiveness) can depend on the functional status of the autonomic system in VS and MCS subjects. This would also add to the existing controversy [35] on whether visual pursuit indicates "automatic" subcortical processing compatible with, but atypical for the VS/UWS [37], or it signals higher order cortical activation and partially recovered consciousness [38].

Independent factors modulating both the brain responsiveness and the autonomic balance are also possible. Residual circadian/ultradian cycles asynchronous among subjects are conceivable. Spontaneous fluctuations and end-effects of neuronal or nonneuronal variables known to individually or collectively account for individual variability [4,39] cannot be excluded in principle no matter how controlled the conditions of observation.
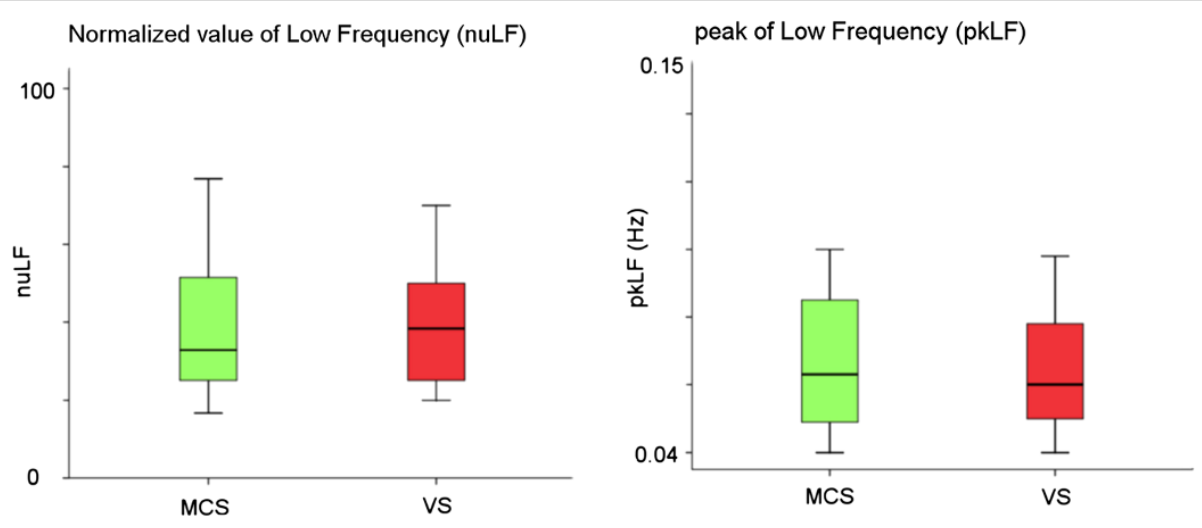

Figure 1 Distribution of the HRV descriptors nuLF and pkLF in the VS/UWS (red) and MCS (green) patients' subgroups at baseline. 


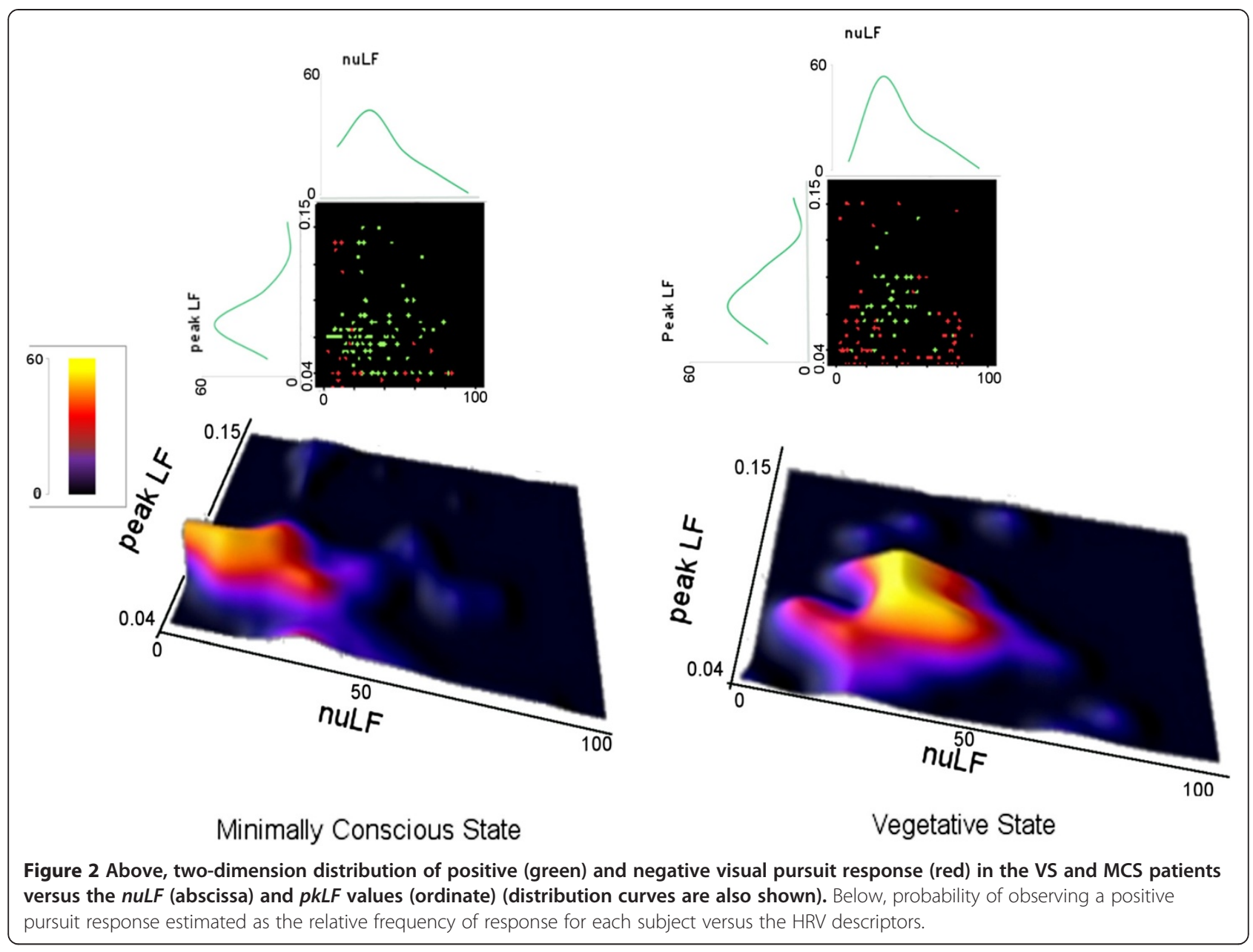

Cohort studies on larger groups of subjects would supplement our observation and provide information on underestimated source of individual variability.

It should nevertheless be noted how the observed incidence of a visual pursuit response in VS/UWS and MCS subjects' subgroups (32.2\% and $79.3 \%$, respectively) and its variability [4] are congruent with the estimated rate of misdiagnosis (30-40\%) [3] between two conditions with somehow unclear boundaries, that share etiology and underlying pathophysiology, but differ as to prognosis, medical, legal, or popular perception of the bioethical issue, allocated resources, healthcare policies, etc. $[1,40]$. Due to its correlation with a positive visual pursuit response, the sympathetic/parasympathetic balance could also qualify as an independent prognostic index to the extent monitoring it can be noninvasive (as in the case

Table 2 Correct classification of response in the visual pursuit

\begin{tabular}{|c|c|c|c|c|c|c|}
\hline & & Correct classification & Accuracy & Balanced accuracy & Sensitivity & Specifity \\
\hline & Training Test & 81.4 & 81.4 & 81.3 & 82.1 & 80.4 \\
\hline \multirow[t]{3}{*}{ General Model } & 10 Fold Cross & 81.4 & 81.4 & 81.3 & 82.1 & 80.4 \\
\hline & Leave One Out & 81 & 79.5 & 77.9 & 84.4 & 71.4 \\
\hline & Training Test & 88.8 & 88.8 & 90.3 & 88.3 & 92.3 \\
\hline \multirow[t]{3}{*}{ MCS Model } & 10 Fold Cross & 87.9 & 87.9 & 89.6 & 87.5 & 91.7 \\
\hline & Leave One Out & 82 & 82.1 & 82.6 & 81.8 & 83.3 \\
\hline & Training Test & 86.9 & 87 & 84.9 & 78.9 & 90.9 \\
\hline \multirow[t]{2}{*}{ SV Model } & 10 Fold Cross & 84.3 & 84.4 & 81.9 & 74.4 & 89.5 \\
\hline & Leave One Out & 77 & 76.9 & 78.4 & 81.8 & 75 \\
\hline
\end{tabular}


of HRV measurements) and reliable. Research on the correlation between the sympathetic/parasympathetic balance and the subject's responsiveness to simple sensory or noxious stimuli is in progress.

\section{Conclusions}

Application of HRV descriptors in the functional characterization of patients with severe disorder of consciousness appears promising $[5-7,13]$ for references. Accurate monitoring of the sympathovagal balance would allow test the visual fixation and eye pursuit response with greater accuracy and have these signs eventually reconsidered as indices of recovered consciousness or predictors of evolution and outcome. By extension, the autonomic system functional state should be re-considered as an independent variable potentially affecting other measures of CNS function of varying complexity, from clinical signs to indices of regional brain activation.

\section{Competing interests}

The authors declare that they have no competing interests.

\section{Authors' contributions}

MDC and FR collected all the data. FR performed all data pre- processing and statistical analyses. All authors were involved in conceiving the study and participated in its design and coordination. All authors contributed to the manuscript from draft to its final version and have agreed on the submitted manuscript. All authors read and approved the final manuscript.

\section{Author details}

${ }^{1} \mathrm{~S}$. Anna Institute and RAN (Research in Advanced Rehabilitation), Crotone, Italy. ${ }^{2}$ Department of Neuroscience, Ophthalmology and Genetics, University of Genova, 3, Largo P. Daneo, 16132 Genova, Italy. ${ }^{3}$ Department of Psychiatry, State University of New York, Stony Brook, NY, USA.

Received: 4 January 2013 Accepted: 14 October 2013

Published: 6 November 2013

\section{References}

1. Laureys S, Celesia GG, Cohadon F, Lavrijsen J, Leòn-Carriòn J, Sannita WG, Sazbon L, Schmutzhard E, Von Wild KR, Zeman A, et al: Unresponsive wakefulness syndrome: a new name for the vegetative state or apallic syndrome. BMC Med 2010, 8:68

2. Giacino JT, Kalmar K: Diagnostic and prognostic guidelines for the vegetative and minimally conscious states. Neuropsychol Rehabil 2005, 15(3-4):166-174

3. Bosco A, Lancioni GE, Olivetti Belardinelli M, Singh NN, O'Reilly MF, Sigafoos J: Vegetative state: efforts to curb misdiagnosis. Cogn Process 2010, 11(1):87-90.

4. Candelieri A, Cortese MD, Dolce G, Riganello F, Sannita WG: Visual Pursuit: Within-Day variability in the severe order of consciousness. J Neurotrauma 2011, 28(10):2013-2017.

5. Riganello F, Candelieri A, Quintieri M, Conforti D, Dolce G: Heart rate variability: An index of brain processing in vegetative state? An artificial intelligence, data mining study. Clin Neurophysiol 2010, 121(12):2024-2034.

6. Riganello F, Candelieri A, Dolce G, Sannita WG: Residual emotional processing in the vegetative state: A scientific issue? Clin Neurophisiol 2011, 122(5):1061-1062.

7. Riganello F, Sannita WG: Residual Brain Processing in the Vegetative State. J Psychophysiol 2009, 23(1):18-26.

8. Monti MM, Vanhaudenhuyse A, Coleman MR, Boly M: Willful Modulation of Brain Activity in Disorders of Consciousness. N Engl J Med 2010, 18(7):579-589.

9. Owen AM, Coleman MR: Functional neuroimaging of the vegetative state. Nat Rev Neurosci 2008, 9:235-243.
10. Appelhans BM, Luecken LJ: Heart rate variability and pain: associations of two interrelated homeostatic processes. Biol Psychol 2008, 77(2):174-182.

11. Berntson GG, Cacioppo JT: Heart rate variability: A neuroscientific perspective for further studies. Card Electrophysiol Rev 1999, 3:279-282.

12. Lombardi F: Clinical implications of present physiological understanding of HRV components. Card Electrophysiol Rev 2002, 6(3):245-249.

13. Riganello F, Dolce G, Sannita WG: Heart rate variability and the central autonomic network in the severe disorder of consciousness. J Rehabil Med 2012, 44(6):495-501.

14. Borer-Alafi N, Gil M, Sazbon L, Korn C: Loewenstein communication scale for the minimally responsive patient. Brain Inj 2002, 16(7):593-609.

15. Hagen C, Malkmus D, Durham P: Levels of cognitive functioning. In Rehabilitation of the head injured adult: comprehensive physical management. Edited by Professional Staff Association of Rancho Los Amigos H. Downey CA: Rancho Los Amigos Hospital Inc; 1987.

16. Rappaport M, Hall KM, Hopkins K, Belleza T, Cope DN: Disability rating scale for severe head trauma: coma to community. Arch Phys Med Rehabil 1982, 63(3):118-123

17. Giacino JT, Kalmar K, Whyte J: The JFK Coma Recovery Scale-Revised: measurement characteristics and diagnostic utility. Arch Phys Med Rehabil 2004, 85(12):2020-2029.

18. Selvara N, Jaryal A, Santhosh J, Deepak KK, Anand S: Assessment of heart rate variability derived from finger-tip photoplethysmography as compared to electrocardiography. J med egin technol 2008, 32(6):479-484.

19. Candelieri A, Cortese MD, Riganello F, Sannita WG: Functional Status And The Eye-Tracking Response. A Data Mining Classification Study in the Vegetative and Minimaly Conscious States. Rome: In Heathlnf Proceeding: 2011; 2011:138-141.

20. Task Force of the European Society of Cardiology the North American Society of Pacing E: Heart Rate Variability Standards of Measurement, Physiological Interpretation, and Clinical Use. Circulation 1996, 93:1043-1065.

21. Niskanen JP, Tarvainen MP, Ranta-aho PO, Karjalainen PA: Software for Advanced HRV Analysis. Com Methods Prog Biomed 2004, 76(1):73-81.

22. Han J, Kamber M: Data mining: concepts and techniques. San Francisco: Department of Computer Science, University of Illinois at Urbana-Champaign. Morgan Kaufmann; 2006.

23. Van Bemmel JH, Munsen MA: Handbook of medical informatics. Berlin: Springer-Verlag; 1997.

24. Burr RL: Interpretation of normalized spectral heart rate variability indices in sleep research: a critical review. Sleep 2007, 30(7):913-919.

25. Nickel P, Nachreiner F: Sensitivity and diagnosticity of the $0.1-\mathrm{Hz}$ component of heart rate variability as an indicator of mental workload. Hum Factors 2003, 45(4):575-590.

26. Peng HC, Long F, Ding C: Feature selection based on mutual information: criteria of max- dependency, max-relevance, and min-redundancy. EEE Transactions on Pattern Analysis and Machine Intelligence 2005, 27(8):1226-1238.

27. Cristianini N, Shawe-Taylor J: An introduction to Support Vector Machines: And Other Kernel-Based Learning Methods. Cambridge, England: Cambridge University Press; 2000.

28. Noble WS: What is a support vector machine? Nat Biotechnol 2006 24(12):1565-1567.

29. Kampouraki A, Nikou C, Manis G: Robustness of Support Vector Machine-based Classification of Heart Rate Signals. New York City, USA: In Proceedings of the 28th IEEE EMBS, Annual International Conference: 2005; 2005.

30. Kohavi R: A study of cross-validation and bootstrap for accuracy estimation and model selection Artificial Intelligence. Proceeding of the fourteenth International Joint Conference 1995, 2(12):1137-1143.

31. Buhmann MD: Radia Bass Function. Acta Numerica 2000, 9:1-38.

32. Crottaz-Herbette $\mathrm{S}$, Menon $\mathrm{V}$ : Where and when the anterior cingulate cortex modulates attentional response: combined fMRI and ERP evidence. J Cogn Neurosci 2006, 18(5):766-780

33. Thayer JF, Lane RD: Claude Bernard and the heart-brain connection: further elaboration of a model of neurovisceral integration. Neurosci Biobehav Rev 2009, 33(2):81-88.

34. Krauzlis RJ: Neuronal activity in the rostral superior colliculus related to the initiation of pursuit and saccadic eye movements. J Neurosci 2003, 23(10):4333-4344

35. Bruno MA, Vanhaudenhuyse A, Schnakers C, Boly M, Gosseries O, Demertzi A, Majerus S, Moonen G, Hustinx R, Laureys S: Visual fixation in the vegetative state: an observational case series PET study. BMC Neurol 2010, 10:35. 
36. Giacino JT: The vegetative and minimally conscious states: consensusbased criteria for establishing diagnosis and prognosis. NeuroRehabilitation 2004, 19(4):293-298.

37. Multi-Society Task Force on PVS: Statement on medical aspects of the persistent vegetative state. N Engl J Med 1994, 330:1499-1508. and 1572-79.

38. Giacino JT, Hirsch J, Schiff N, Laureys S: Functional neuroimaging applications for assessment and rehabilitation planning in patients with disorders of consciousness. Arch Phys Med Rehabil 2006, 87(12 Suppl 2):S67-S76.

39. Sannita WG: Individual variability, end-point effects and possible biases in electrophysiological research. Clin Neurophysiol 2006, 117(12):2569-2583.

40. Racine E, Amaram R, Seidler M, Karczewska M, Illes J: Media coverage of the persistent vegetative state and end-of-life decision-making. Neurology 2008, 71(13):1027-1032.

doi:10.1186/1471-2377-13-164

Cite this article as: Riganello et al.: Visual pursuit response in the severe disorder of consciousness: modulation by the central autonomic system and a predictive model. BMC Neurology 2013 13:164.

\section{Submit your next manuscript to BioMed Central and take full advantage of:}

- Convenient online submission

- Thorough peer review

- No space constraints or color figure charges

- Immediate publication on acceptance

- Inclusion in PubMed, CAS, Scopus and Google Scholar

- Research which is freely available for redistribution 